ESAIM: PROCEEDINGS AND SURVEYS, Vol. ?, 2019, 1-10

Editors: Will be set by the publisher

\title{
GRAVITATIONAL WAVES: WHERE WE ARE, WHERE WE GO
}

\author{
Fulvio Ricci ${ }^{1}$
}

\begin{abstract}
We review the present status of the Gravitational wave detectors on the Earth, focusing the attention on the present innovations and the longer term perspectives to improve their sensitivity. Then we conclude mentioning few potential searches of new Physics phenomena to be performed with these detectors and those of the third generation.
\end{abstract}

\section{THE DETECTORS STATUS}

In 2019-20120 the two Advanced gravitational-wave detectors, LIGO in USA, Virgo in Italy and KAGRA in Japan, will start to take data in coincidence. This worldwide network will be capable to improve the sky location of the transient GW events as neutron start - neutron star (BNS) or black-hole-neutron star (BHNS) coalescences, providing crucial information to the astronomy community and triggering the multi-messenger observations.

The last data taking of LIGO and Virgo ended at the end of August 2017. Since this time several improvements have been implemented on these detectors.

In the LIGO sites the main hardware changes invested the vacuum system. The main actions have been the replacement of the mirrors acting as test masses and the signal recycling, the installation of the squeezing light benches in both sites. In addition an extended campaign to fight against the stray light has been pursued and the preliminary results seem very promising.

Virgo changed is laser source and installed the squeezing bench built by the group of Albert Einstein Institute of the Max Planck in Hannover. The main change is marked by the re-introduction of the monolithic suspensions of the mirrors. These suspensions made of thin fused silica wires were used during the run of 2011 with the 22 $\mathrm{kg}$ mirrors. In 2017 the mirrors of $42 \mathrm{~kg}$ were suspended by means of metallic wires, more reliable but with a higher contribution of the suspension thermal noise limit.

At present the three instruments are in a commissioning phase and the start of the science run, O3, is planned for April 2019. The scientific target is to achieve sensitivities curves similar to those reported in the article [1] where the collaborations LIGO, Virgo and KAGRA predict the detector performances in the following years .

The KAGRA interferometer has the unique peculiarities to be installed in an underground area and the test mass mirrors are cooled at $20 \mathrm{~K}$. The installation of the interferometer started in 2015 the first test was carried on a $3 \mathrm{~km}$ Michelson interferometer with room temperature mirrors. Then the installation progressed rapidly in the years 2017 and 2018 with the cryogenic payloads equipped with the end mirrors made of sapphire cooled successfully down to $18 \mathrm{~K}$. The KAGRA international collaboration is working hard to join LIGO and VIRGO in the second part of the O3 data taking.

\footnotetext{
${ }^{1}$ Dipartimento di Fisica, Università di Roma Sapienza \& INFN - Sezione di Roma,

Piazza A. Moro 2, 00185 Roma, Italy
} 

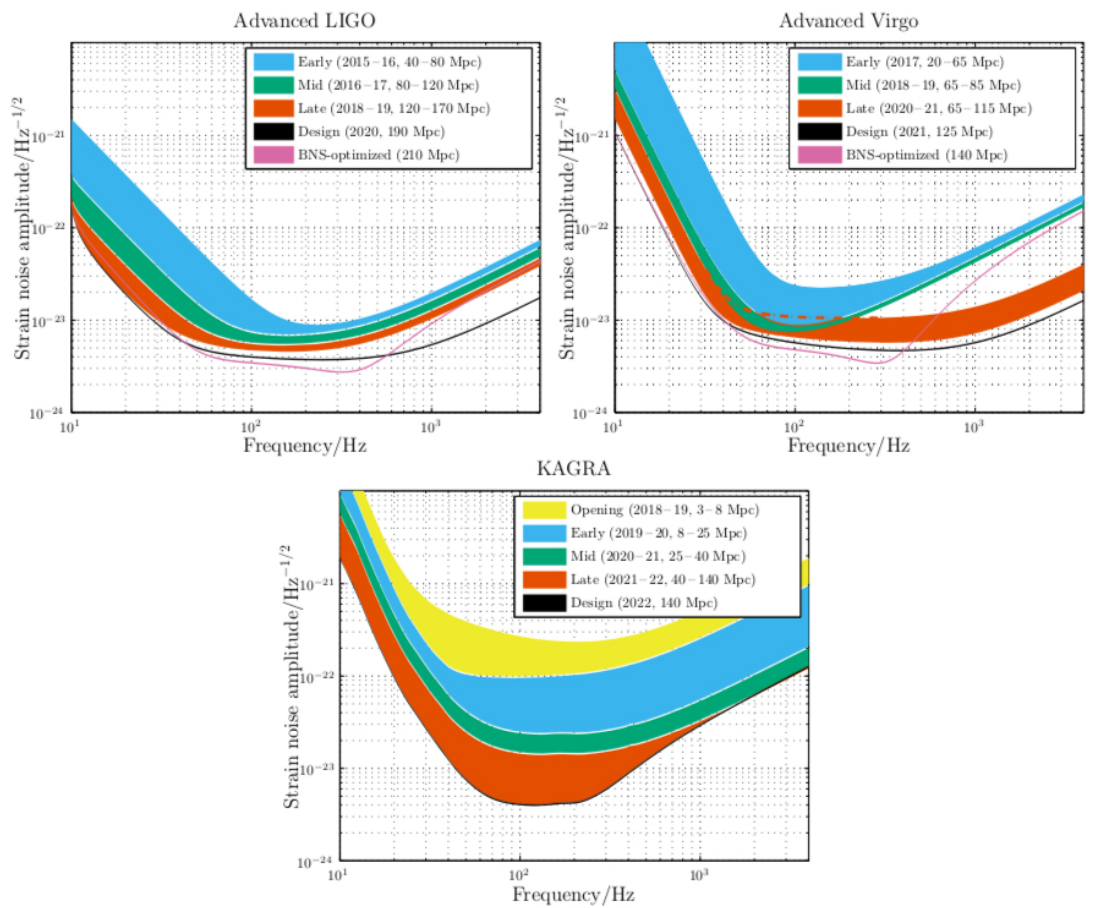

FIGURE 1. The target strain sensitivities of LIGO, Virgo and KAGRA as a function of frequency during the following data taking. The binary neutron star (BNS) range is the average distance in $\mathrm{Mpc}$, to which these signals could be detected. The figure is extracted form the reference [1]

The next decade the gravitational community should converge toward the constructions of new detectors. The network of the advanced detectors, enriched by the inclusion of INDIGO, an other interferometer of the second generation whose construction is just started in India, should achieve the sensitivity values whose limitations are essentially related to the location and the space availability of the present facilities.

Within the international scientific community various initiatives are being carried out to study and implement third generation detectors (3G) able to expand by 1000 times the volume of the universe explored. To achieve this goal it is necessary a significant leap in the sensitivity of the instruments, which can be pursued on one hand by introducing new technological solutions, on the other by building a new infrastructure with different characteristics from the current ones.

European research agencies operating in the gravitational wave sector, brought together in the European APPEC Consortium, have started a preparatory work to present the application for a large research infrastructure for Earth based gravitational astronomy.

The present proposals, as for example the European project Einstein Telescope (ET) targets three crucial innovations, two of them studied already in KAGRA:

- the use of cryogenics to cool down the test mass 
- the installation of the interferometer in an underground laboratory (or at lest the vacuum chambers hosting the test masses)

- the increase of the arm length of the interferometer

Italy has played a leading role in the study of gravitational waves and detector development thanks to the long-term support of INFN. This study produced significant scientific and technological achievements for Virgo, built in collaboration with of the French CNRS. Italy played a leading role also in the context of the ET design study, the project financed by the European Union in the Framework Program 7. On the base of this legacy INFN, with the full support of the local political and academic authorities, is presenting its candidature proposing to host the new detector at the Sos Enattos mine in Sardinia, an ideal place for the construction of the new infrastructure, compatible with the requirements of very low seismic and anthropic noise. The first stage has been accomplished already by Regional Government of Sardinia, which assigned to the University of Sassari a budget for the construction of the SAR-GRAV a research infrastructure in the Sos Enattos mine, a low noise laboratory devoted to host small scale gravity experiments.

New detectors imply also new technological solutions developed thanks to a robust R \& D effort to be started from now days. . The R\&D program paving the way for the realisation of the next generation of instruments, should be focused on some crucial technologies, as

- the improvement of the VIRGO seismic attenuation system, to improve the low frequency sensitivity and to maintain the INFN historic leadership in this field,

- the design, construction and test of a cryogenic payload,

- the development of innovative frequency dependent squeezing techniques for reducing quantum noise,

- the improvement of the (optical and mechanical) losses of the mirrors coatings, to reduce thermal noise.

\section{Scientific targets of the Gravitational Wave Physics}

The direct detection of GW signals, started on September 14th, 2015 with the observation of the coalescence of a binary black hole system $(\mathrm{BBH})$ and then followed by several other observations confirmed the long-standing prediction of Albert Einsteins general theory of relativity and opened up a completely new way of observing Nature. The detected signals confirmed the existence of black holes with masses larger than $20 M_{o}$ dot, but at the same time new questions arose: how many are the black holes in the Universe and what is their size? How are they formed? Since the stellar wind depends on metallicity, the question is how metallicity environment influences the formation?

Now days two models on the binary black hole formation are dominant. The first one hypotheses that we are dealing with two object formed and exploded at the same time from two stars with similar spins oriented in the same direction. The second one assumes that the black holes in a stellar cluster sink to the center of the cluster and pair up spin randomly oriented. The observation of several BBH events should permit to disentangle the two hypothesis on the base of the observed spin distribution In addition the detailed reconstruction of the $\mathrm{BH}$ mass function distribution can tell us about the Universe formation. Other questions are open. Do miniature black holes exist? In the case of a positive observation we can conclude that they have formed immediately after the Big Bang. Rapidly expanding space may have squeezed some regions into tiny, dense black holes less massive than the sun. Under a simplified hypothesis of a uniform distribution of BBH creation on the universe history, with a $3 \mathrm{G}$ detector we expect to observe up to $10^{5} y^{-1} \mathrm{BBH}$ with an impressive signal to noise ratio (SNR) of the order of $10^{4}$ for rare events. The population study will be influenced by the detector sensitivity and the achievable SNR. GW signal amplitude depends on $\mathcal{M}^{5 / 2}$, where $\mathcal{M}=$ the chirp mass defined as $\mathcal{M}=\left(m_{1} m_{2}\right)^{3 / 5} /\left(m_{1}+m_{2}\right)^{1 / 5}$ Higher $\mathcal{M}$ should imply easier detection of the GW signal, but on the other hand the signal duration decreases with $M_{t o t}=m_{1}+m_{2}$ tends to decrease the SNR. In addition we note that too massive systems emit GW signals at frequency out of the bandwidth of the Earth detectors.

GW is the new tool not just for funding a new chapter of Astronomy, we will rely on this new probe to expand our comprehension of the fundamental laws of the Physics. It is widely spread the believe that General Relativity (GR) is incomplete because it is at odds with quantum mechanics. For this reason the GR tests in 
the strong field regime are crucial and the detector network can perform tests also via the observation of the polarisation states of GW.

$\mathrm{BH}$ is the main example of and object dominated by extreme gravity and GW seems to be the best way to probe its properties. The spectrum of Quasi Normal Modes (QNM) is characterised only by the BH mass and angular momentum. The detection of a few modes from the ringdown signal can allow for precision measurements of the $\mathrm{BH}$ mass and spin [2]. In addition the detection of higher multipole moments can be used to perform null-hypothesis tests of the no-hair theorems of general relativity. 3G and space detectors as
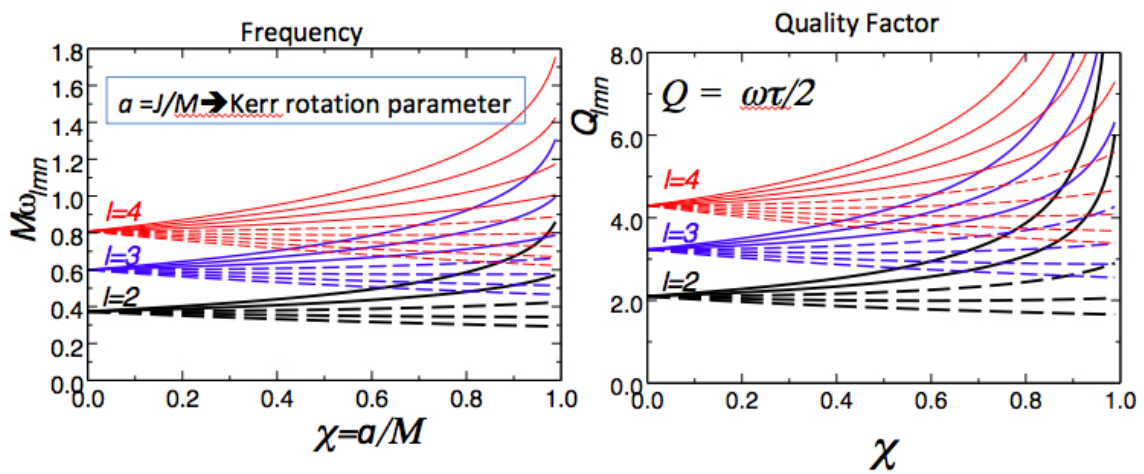

Figure 2. Frequencies and quality factors for the fundamental modes with $l=2,3,4$ and different values of $\mathrm{m}$. Solid lines refer to $m=l, . ., l$ (from top to bottom), the dotted line to $\mathrm{m}$ $=0$, and dashed lines refer to $m=1, . ., l$ (from top to bottom). Quality factors for the higher overtones are lower than the ones we display here.

LISA are ideal instruments for the ring-down observation of the BBH post merger phase. High-SNR events will give individual tests of GR, while the combination of many events collected with both advanced and $3 \mathrm{G}$ interferometers provide stronger constraints on GR.

The Einstein theory of Gravitation predicts that whenever crosses the BH event horizon the information it contains is lost, in contradiction with the expectations of quantum mechanics for which information can neither be created nor destroyed. The existence of Hawking radiation is directly related to this issue, because if in the evaporation the $\mathrm{BH}$ disappears all of the information into them flow. The detection of GW due to the excitation of the $\mathrm{BH}$ quasi normal modes, and in general the detection of the part of the BBH signal after the merger phase at high SNR, can tell us more about the BH nature and the properties of its horizon. The existence of echoed signals could suggest that BH are not bounded by a GR horizon but instead by a quantum-mechanical Planck-scale structure. The time delay between the echoes will be connected to the exotic compact object resembling a black hole and it should depend on its compactness, while the decay and shape of each pulse should encodes its reflective properties [4], [3].

In the future GW can probe the existence of the most exotic objects emerging from the complex mathematical framework of the GR equations. Again looking at the BH quasi normal modes we can try to disentangle the existence of classical BH from a more exotic traversing wormhole. In fact, it has been shown, under very specific assumptions [3], that a point particle, which plunges radially into a wormhole, when it crosses each of the light rings curves, it excites QNM characteristic modes trapped between the light-ring potential wells. In this very specific case the point particle plunges radially and emerges in another universe. When the particle crosses each of the light rings curves, it excites QNM characteristic modes trapped between the light-ring potential wells. The wormhole GW signal is dephased to the respect of that of a classical BH, due to the light travel time from 


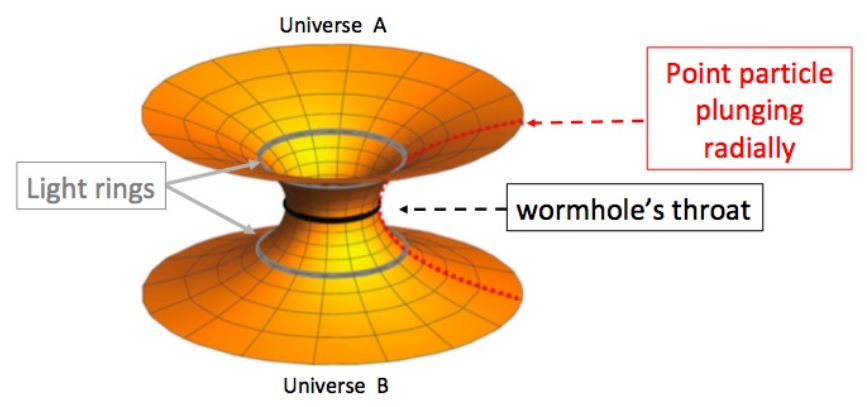

Figure 3

the throat to the light ring. To get evidence of the waveform difference will be a challenge, but if we succeed, it will represent a giant step forward in the exploration of our universe.

In conclusion, the future of the GW physics will be rich of new surprises and conundrums to be solved.

\section{REFERENCES}

[1] Abbott B P. et al., Living Reviews in Relativity December, 21:3 (2018)

[2] Berti E.,Cardoso V., Starinets O., Classical and Quantum Gravity, 26 (16) (2009)

[3] Cardoso V. , Franzin E., Pani P., PRL 116, 171101 (2016)

[4] Mark Z., Zimmerman A., Du S. M., Chen Y., PRD 96, 084002 (2017) 